\title{
Research of Point to Line on Practical Teaching Process in Electronic and Information Engineering Specialty
}

\author{
Ming Zhao, Yuru Zhang, Xiaodong Su, Hui Li, Hao Jin, Yun Li \\ School of Computer and Information Engineering, Harbin University of Commerce, Harbin, China \\ hrbcu_lh@163.com
}

Keywords: Electronic and Information Engineering; Practice Teaching; Teaching Reform; Proteus

\begin{abstract}
This paper explores the method of Point to Line on practical teaching reformation in electronic and information engineering Specialty. The common points have been found to connect relevant practice teaching of the course. It can not only help the students to link up theory knowledge, but also further inspire the enthusiasm and motivation of scholars. Based on Proteus, this paper introduces a case of using waveform generator as a point to connect the practical teaching of several related courses of electronic information engineering Specialty. This teaching method has been proved to ensure the continuity of knowledge, which does not affect the course independence and expansibility. Above all, the method can motivate students' interests in learning and exploration.
\end{abstract}

\section{Introduction}

Electronic and information engineering specialty aims to train students to gain the ability of electronic product design, research and development, signal and information processing, and information system integration. Nowadays, with the rapid development of science and technology, electronic and information technology develops rapidly and new products emerge endlessly. In order to improve the innovation spirit and entrepreneurial ability, teaching reformation must be carried out, and the very important step is the practical teaching reformation. According to the specialty characteristics of electronic and information engineering, it is more significant to develop a perfect practical teaching system for the personnel training. On the premise of the reasonable training plan formulation, we classify the courses in training scheme and find relevant course practice teaching of the common points, from the point to the line. The key point in the practice of teaching reformation is to deepen the related knowledge in practice teaching [1].

\section{Sort Out the Professional Curriculum Structure and Practical Teaching Links}

There are three modules of the electronic and information engineering specialty core courses:

\subsection{Basic course module}

Basic course modules mainly include the common courses such as "Circuit Analysis", "Analog Electronics Technology", and "Digital Logic”, which are the basis for learning subsequent courses.

\subsection{Information and communication engineering course module}

Information and communication engineering course module mainly includes "Signals and Systems", "Communication Clectronic Circuit", "Random Signal Processing”, "Information Theory and Coding”, "Digital Signal Processing” and "Digital Image Pocessing”.

\subsection{Control science and engineering course module}

Control science and engineering course module mainly includes "Application of Single-Chip Microcomputer”, “Sensor Technology”, “Embedded Systems”, “Wireless Sensor Network” and “Automatic Control Theory” [2]. 
Through sorting out the curriculum structure, we can research on the relevant specialized courses from point to line practice teaching mode. Firstly Proteus software has been introduced in the process of practice teaching, and dynamic simulation teaching have been improved in "Circuit Analysis", "Analog Electronics Technology", and "Digital Logic". Theoretical calculation and actual running results are pocessed to improve the teaching efficiency effectively, and it is the point to line and the practice teaching mode of study of the important tools. With the introduction of $C$ and MATLAB language, he practice teaching of other courses form from point to the line of practice teaching mode of research provides a powerful tool. Based on waveform generator, "Circuit Analysis”, “Analog Electronics Technology", "Digital Logic" and "Application of Single-Chip Microcomputer" have been in series. The practice teaching mode of point to the line has been studied based on Proteus [3].

\section{Practical teaching model with waveform generator as a point}

Based on waveform generator as a point, Practical teaching model would be constituted by "Circuit Analysis”, “Analog Electronics Technology”, "Digital Logic”, "SOPC” and "Application of Single-Chip Microcomputer”, as shown in Fig.1.

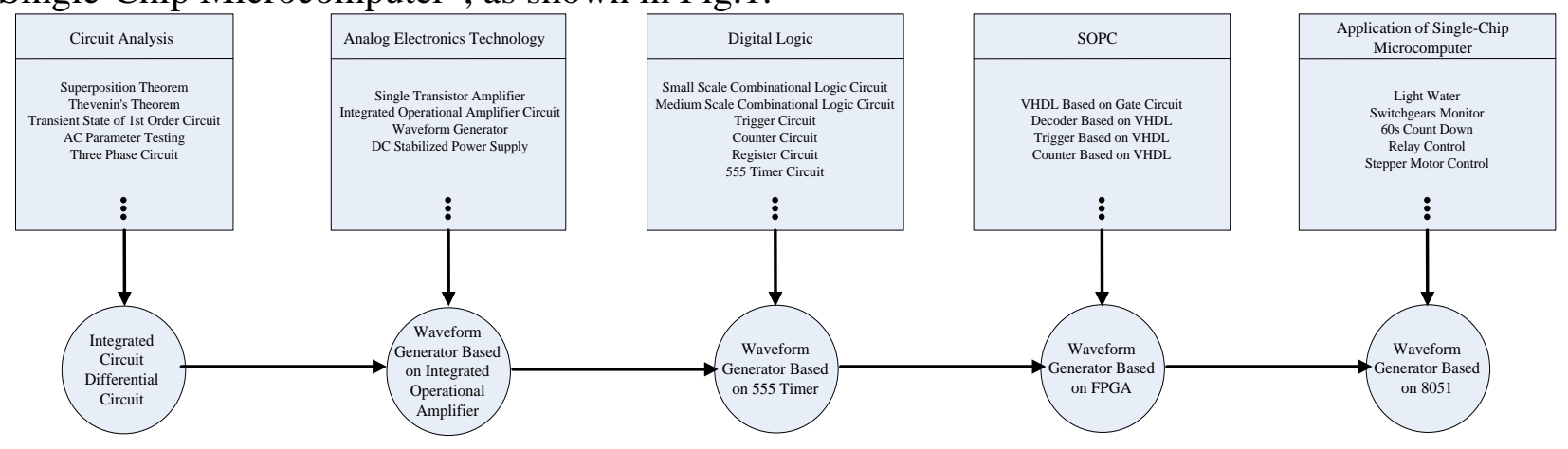

Figure.1 Structure of practical teaching model with waveform generator as a point

"Circuit Analysis" is the first professional basic course for our students. The teaching model which simulates first and tests then with introducing Proteus software, can not only simulates dynamicly of important and difficult problems of course in class, but also can improves the efficiency of experiments with this method. The common point of "teaching mode from point to line" starts from the transient process of first-order circuit in "Circuit Analysis". The time constant has been transformd with the value of RC, and the output of the circuit presents different waveform. The process of square wave to triangle wave is emphasized by simulation with Proteus. The simplest waveform generator will be proposed in "Analog Electronics Technology" and "Digital Logic" course. The operation situation how square wave transforms into triangle wave will be observed in the laboratory, and the relevant knowledge will be emphasizes in the realization of waveform transformation.

Fig. 2 is the simulation circuit diagram of square waves convertes into triangular waves with integrated circuit in "Circuit Analysis".
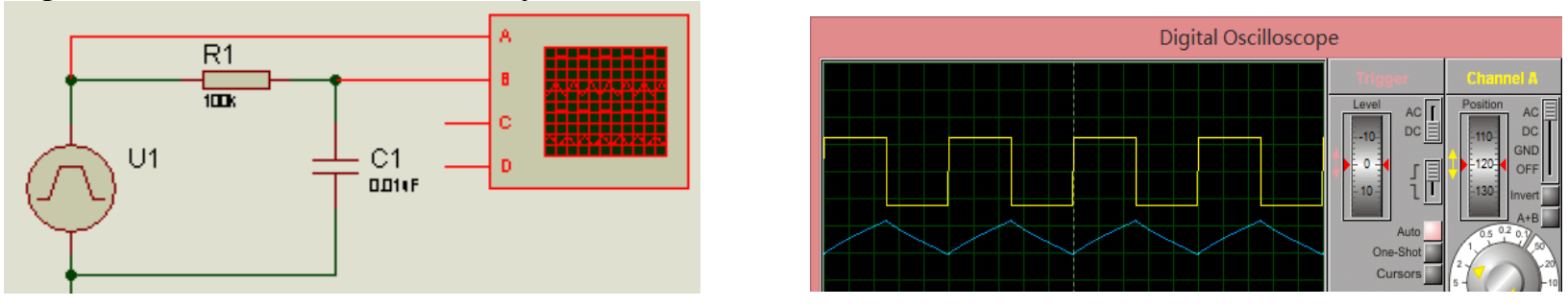

Figure.2 Integrated circuit and waveform figure

In “Analog Electronics Technology" course, waveform generator experiment based on the integrated operational amplifier has been set, and sine wave, square wave and triangular wave would been produced by $\mu \mathrm{A} 741[4]$. Fig.3 is the waveform figure of waveform generator based on $\mu \mathrm{A} 741$. 

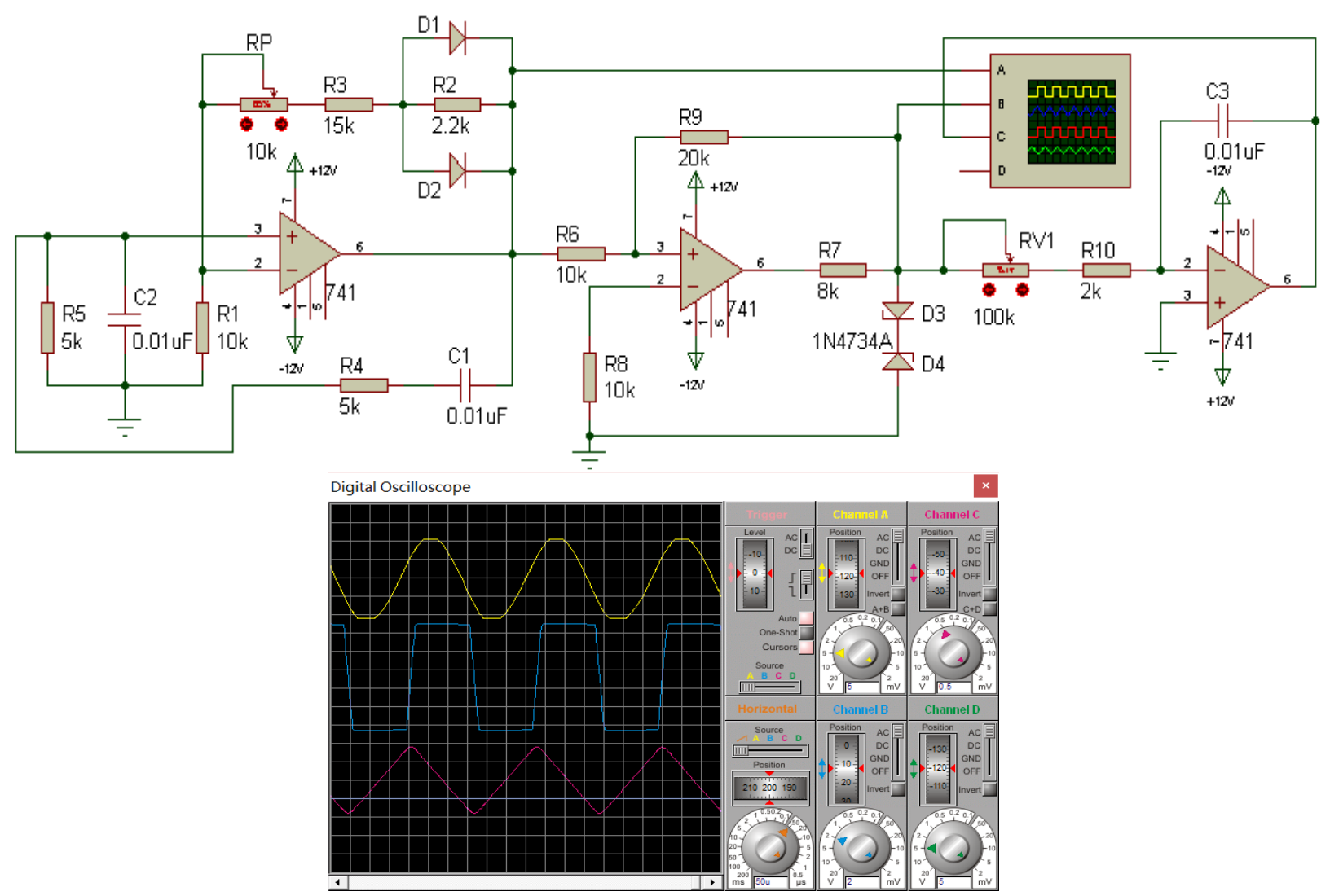

Figure.3 Circuit and waveform figure of waveform generator based on $\mu \mathrm{A} 741$

Students can also realize the function of waveform generator by using 8038 and 555 timers through consulting data, and 555 timers are explained in "Digital Logic” course. The students' ability to scientific research and exploration should be exercised by this practice teaching process.

The experiment of waveform generator would be set in "SOPC" and "Application of Single-Chip Microcomputer". This circuit should be realized for waveform as sine wave, square wave, triangular wave and sawtooth wave with FPGA. Fig. 4 is the output waveform figure of triangular wave as shown in oscilloscope.
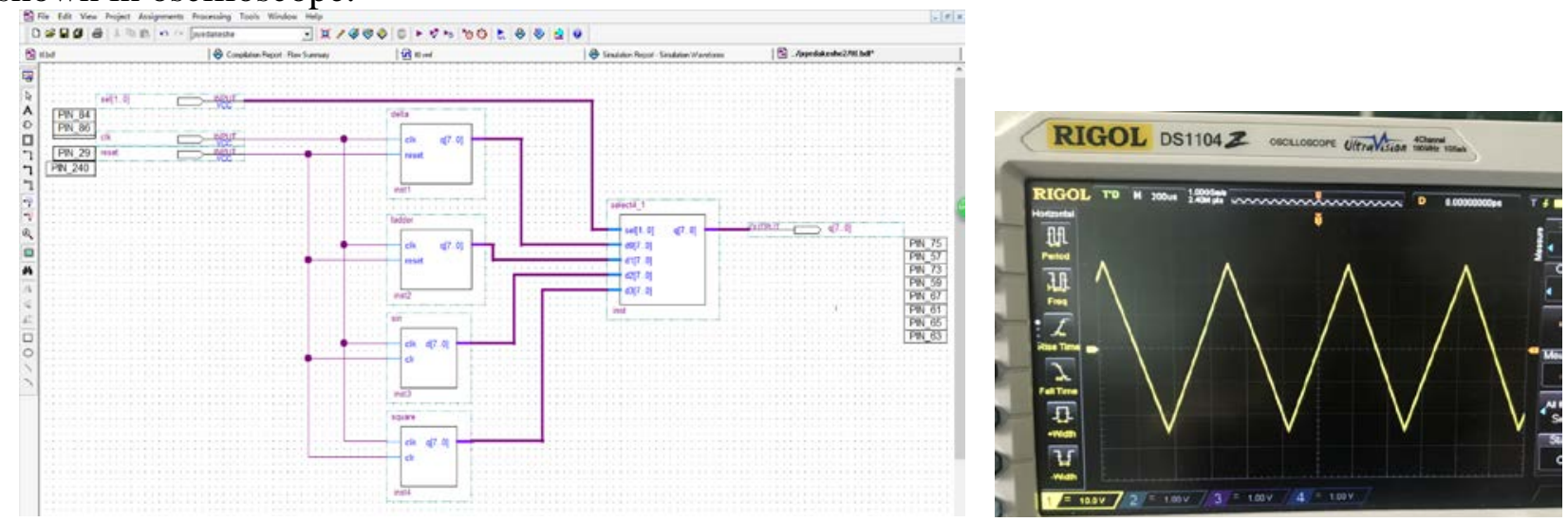

Figure.4 Circuit of waveform generator based on FPGA and sawtooth wave figure

Fig. 5 is circuit of unipolarity waveform generator based on AT89C51 and sawtooth wave figure, and the circuit of bipolar waveform based on AT89C51 also can be realized. [5] the output of some waveform will be realized with software program, and frequency and amplitude will be adjust.

The common point should be determined by the line connect with practice teaching, and by the point with waveform transformation and waveform generator. According to the characteristics of each course, the integrity and independence of courses would be guaranteed. 

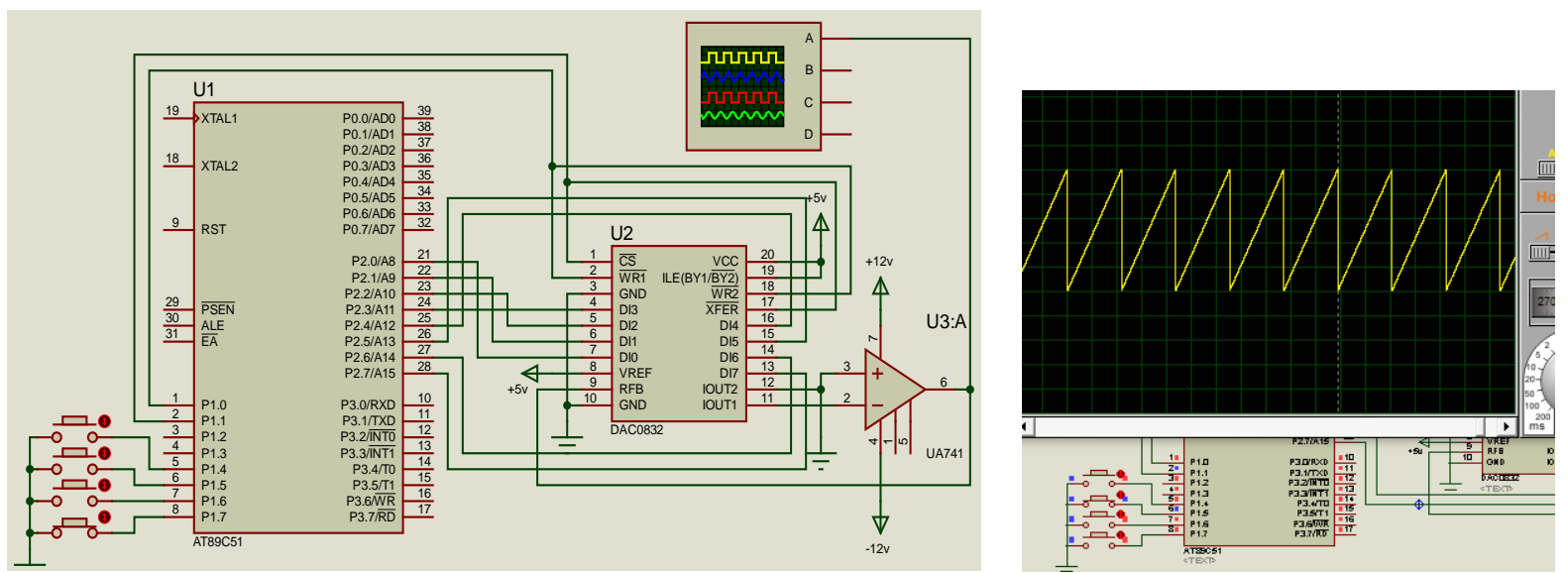

Figure.5 Circuit of unipolarity waveform generator based on AT89C51 and sawtooth wave figure

\section{Summary}

The common point should be determined by the line connect with practice teaching, and by the point with waveform transformation and waveform generator. According to the characteristics of each course, the integrity and independence of courses would be guaranteed.

\section{Acknowledgements}

This work was financially supported by Cooperative and Cooperative Education Project of Ministry of Education of the People's Republic of China (201601023031, 201702029029, 201702064010), Teaching Reform Research Projects of Heilongjiang Education Department (SJGY20170055, SJGY20170075), Teaching Reform Research Projects of Harbin University of Commerce (SJXM2018A004, HSDJY014), Natural Science Foundation of Heilongjiang Province of China (F2015045), University Nursing Program for Young Scholars with Creative Talents in Heilongjiang Province (UNPYSCT-2016063) and Science Foundation of Harbin Commerce University (17XN060).

\section{References}

[1] Dai Fen, Wang Weixing, i Zhen, Sun Daozong, Deng Xiaoling, Jiang Sheng. Research on the Cultivation of Undergraduate Talents in Electronic and Information Engineering Specialty, China Electronics Education No.1 (2016)

[2] Yan Gaizhen, Xu Chaoshen, Li Shuangxi. Exploration on the Engineering Education Oriented Electronic Information Specialty Construction. academic research.

[3] LIU Chun-ling, HE Ting, LU Hai-tian, WANG Chun-wu. Construction and practice of the course groups with different orientations in electronic information engineering major. Journal of Science of Teachers College and University (2018)

[4] Hu Zhongyu, Yue Qiang, Ren Jie, Pu. Innovation on teaching of electrical and electronic courses based on proteus simulation. Journal of Science of Teachers College and University (2018)

[5] LIU Chun-ling, HE Ting, LU Hai-tian, WANG Chun-wu. Construction and practice of the course groups with different orientations in electronic information engineering major. Cuiping Expermental Technology and Management, Vol.33 No.4 (2016) 